\title{
MODELING OF CHEMICAL AND MIXING EFFECTS ON METHANE AUTOIGNITION UNDER DIRECT-INJECTION, STRATIFIED CHARGED CONDITIONS
}

\author{
S. HONG, ${ }^{1}$ M. S. WOOLDRIDGE ${ }^{2}$ AND D. N. ASSANIS ${ }^{1}$ \\ ${ }^{1}$ Department of Mechanical Engineering \\ University of Michigan \\ 1231 Beal Ave. \\ Ann Arbor, MI 48109, USA \\ ${ }^{2}$ Department of Mechanical Engineering \\ University of Michigan \\ 2350 Hayward St. \\ Ann Arbor, MI 48109, USA
}

\begin{abstract}
Fundamental experiments and direct numerical simulations indicate that reaction rates during the ignition delay of direct-injection, stratified charge mixtures are determined by both chemical reaction and mixing rates. Consequently, the commonly used, purely chemistry-dependent approach for predicting ignition is no longer valid when the mixing rate limits the reaction rates. This work proposes an improved approach for predicting the reaction rates during ignition delay which accounts for both chemistry and mixing. Initially, reaction rates are determined using only the chemical reaction rate. Subsequently, a transition is made to the use of a modified eddy dissipation concept in which reaction rates are determined based on interaction between chemical reaction rates and mixing rates. To illustrate and validate the model, compression ignition under stratified charged conditions is considered for methane/air mixtures with temperatures ranging from 1200 to $1500 \mathrm{~K}$. The predictions agree well with the experimental results qualitatively as well as quantitatively. Furthermore, by comparing our approach against a chemistry-only model, conditions where mixing plays an important role in predicting reaction rates during the ignition period are identified.
\end{abstract}

\section{Introduction}

Direct-injection, stratified charge (DISC) engines using methane promise thermal efficiencies comparable with those attained by high-compression-ratio, unthrottled diesel engines while maintaining the smoke-free operation of premixed-charge spark-ignited engines and producing much lower $\mathrm{NO}_{x}$ emissions $[1,2]$. Nevertheless, the primary challenge in operating such compression-ignition DISC engines is the low ignitability of methane. The ignition process is very complex and involves many physical and chemical steps [3-10]. The physical or mixing delay is controlled by the rates of fuel injection and fuel/ air mixing. The chemical delay is due to precombustion reactions of fuel, air, and residual species leading to autoignition.

Ignition models typically used in engine simulations [1,11-13] predict reaction rates during the ignition period based on a purely chemistry-dependent approach under the assumption of small Damköhler number. Such chemistry-dependent models can be used to predict reasonable values for ignition delay provided that detailed reaction schemes [1] or simplified schemes tuned for certain applications [11-13] are employed. However, investigations conducted on simple counterflow or coflow flame configurations [3-10] have shown that this approach is compromised when turbulent mixing plays an important role in determining reaction rates during the ignition period.

In such fundamental experiments and direct numerical simulation studies $[7,9,10]$, ignition of fuel/ air which is premixed during the induction time is quasi-laminar, and the reaction rate during this period depends on the mean species concentration of the prepared mixture and the local mean temperature. Subsequently, the diffusion flame propagates according to the rate that fuel/air mixing is controlled by turbulent properties such as strain rate. In particular, the effect of turbulence on ignition is important in the diffusion phase since mixing occurs in the thin mixing layer which can be distorted by the turbulence. These studies suggest ignition cannot be defined as only a chemistry-dependent phenomenon and physical mixing should be included in the prediction of ignition delay for certain operating conditions. Nevertheless, current large-scale engine simulation codes (e.g., KIVA [14]) do not provide 
sufficiently fine resolution to calculate strain rate due to the large computational costs involved.

Kong and Reitz [15] have proposed a practical approach to account for turbulent effects on prediction of ignition by proposing a new reaction rate which incorporates the effects of both chemical kinetics and turbulent mixing through characteristic time scales. The turbulent time scale was defined as the time for eddy breakup, while the kinetic time scale was estimated as the time needed for a species to reach equilibrium under perfectly mixed conditions. However, two simplifying assumptions were imposed in determining the kinetic time scales: (1) fuel concentration was assumed to be zero at equilibrium; (2) the kinetic time scale for all species was the same as that of selected reference species (where the reference species change from fuel to combustion products as combustion progressed). The first assumption leads to relatively long kinetic times, while the choice of the reference species in the second assumption is somewhat arbitrary.

In the present study, an improved model is proposed to simultaneously account for the effects of chemistry and mixing on ignition delay. Our model comprises a combination of the chemistry-only approach, used during the induction time, and a modified eddy dissipation concept (EDC), used subsequently. The modified EDC model is used to predict the reaction rate on the basis of the interaction between chemical and mixing rates. Detailed chemistry is used throughout the prediction of ignition delay in this study because the minimum ignition energy and induction time cannot be well predicted by simplified chemistry $[1,16,17]$.

A transition model was also developed to predict ignition when the local computational volume is between the chemistry-only and chemistry-mixing regimes. The transition model is based on the concepts of branched-chain explosion [18-21] and thermal explosion [18-20]. Branched-chain explosion defines the ignition time as the time required to achieve radical growth or appreciable reactant depletion, while thermal explosion defines the ignition time as the time to obtain thermal runaway. The transition from the chemistry-only model to the mixing-chemistry model is determined using a transition parameter which accounts for both types of explosion phenomena by assuming radical growth and/or thermal runaway are characterized as proportional to fuel consumption.

The effects of mixing on ignition are investigated by comparing results obtained using a typical chemistry-only ignition model and the proposed combined chemistry-only and chemistry-mixing model. The effect of the transition parameter on prediction of ignition delay is also examined.

\section{Modeling}

The computational platform used was the KIVA3V code [14], modified at the University of Michigan to simulate gaseous injection [22] and to link with CHEMKIN-II [23] and CEA [24]. A detailed chemical kinetic mechanism, a reduced version of GRIMech 1.2 [25], consisting of 24 species and 104 elementary reactions was used to simulate methane gas ignition and combustion. The stiff ODE solver LSODE [26] was used to integrate the source terms in the species equations.

Two approaches are used to predict ignition delay. In the first approach, a chemistry-only ignition model (referred to as model 1) predicts the local reaction rate using only chemical kinetics during the ignition delay, a common approach used in the literature [1,11-13]. The improved approach proposed in this study consists of using either the chemistryonly ignition model or a modified EDC model to determine the local reaction rate. Which model (chemistry only or chemistry and mixing) is used depends on the local value of the transition parameter, discussed further below. The results of this approach are referred to as model 2 . The chemistry-only ignition model used in model 2 is identical with that used in model 1. The modified EDC model is activated only when predefined fuel consumption for radical growth is achieved.

\section{Chemistry-Dependent Ignition Model}

The chemistry-only ignition model [1] calculates reaction rates using detailed chemistry. In this ignition model, it is assumed that the chemical time scales are much larger than the flow time scales during ignition delay, and the turbulent effects are negligible. Based on these assumptions, the reaction rates are calculated using elementary rate coefficients, the mean temperature, and mean species concentrations. The net rate of creation or destruction of species $m$ is determined via

$$
\begin{gathered}
\dot{\omega}_{m}=\dot{C}_{m}-\dot{D}_{m} \\
\dot{C}_{m}=\sum_{i=1}^{N} v_{m i}^{\prime} k_{r_{i}} \prod_{j=1}^{M}\left[X_{j}\right]^{v \prime_{j i}}+\sum_{i=1}^{N} \\
v_{m i}^{\prime \prime} k_{f_{i}} \prod_{j=1}^{M}\left[X_{j}\right]^{v{ }_{j i}} \\
\dot{D}_{m}=\sum_{i=1}^{N} v_{m i}^{\prime} k_{f_{i}} \prod_{j=1}^{M}\left[X_{j}\right]^{v{ }_{j i}}+\sum_{i=1}^{N} \\
v_{m i}^{\prime \prime} k_{r_{i}} \prod_{j=1}^{M}\left[X_{j}\right]^{v \prime_{j i}}
\end{gathered}
$$

where $v^{\prime}{ }_{j i}$ and $v^{\prime \prime}{ }_{j i}$ are the stoichiometric coefficients, $k_{\mathrm{f}_{i}}$ and $k_{\mathrm{r}_{i}}$ are the forward and reverse rate constants for the reaction $i$, and $\left[X_{j}\right]$ is the molar concentration of the species $j$. 


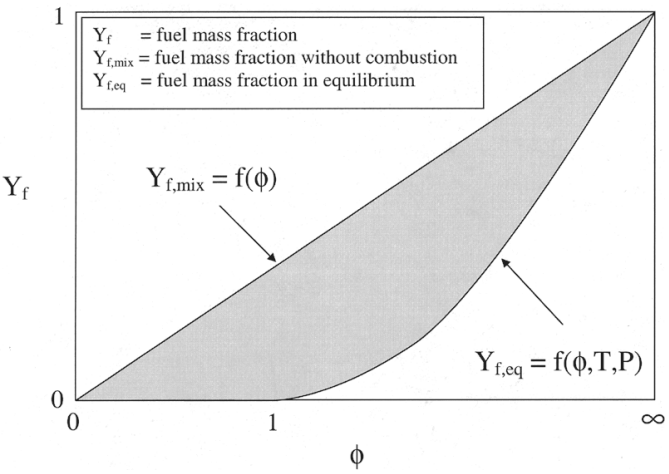

FIG. 1. Schematic indicating the mass fraction of methane fuel as a function of equivalence ratio for the two limiting extremes of frozen chemistry and equilibrium conditions.

\section{Modified EDC Model}

The original EDC model [27] is based on a steady, perfectly stirred reactor concept, which identifies a reaction zone in the intermittently distributed turbulent fine structures where chemical reactions take place. The fine structures are characterized using the length and time scales from the turbulence model, and mixing in the fine structures is assumed to be fast. Outside the reactor are surroundings from which the mass transfer of species takes place. The reaction rates within the fine structures are related to average reaction rates for the bulk fluid, which are applied in the time-averaged transport equations. Our basic concept is to use the EDC model to represent the molecular mixing at the subgrid level where reactions occur in a small-scale mixing zone. Therefore, the EDC model is applicable for the both non-premixed and premixed combustion cases (including early injection conditions), and the overall coupled chemistry/mixing modeling approach is valid for partially premixed and non-premixed conditions.

In order to be applicable to non-steady systems such as engines, it is necessary to add transient terms to the EDC model. The governing equation for the mass fraction of species $m$ in the fine structures is

$$
\frac{\mathrm{d} Y_{m}^{*}}{\mathrm{~d} t}=\frac{\dot{m}^{*}}{1-\chi \gamma^{*}}\left(\bar{Y}_{m}-Y_{m}^{*}\right)+\frac{\dot{\omega}_{m}^{*} W_{m}}{\rho^{*}}
$$

where $\chi$ is the fraction of fine structures which reacts, $\gamma^{*}$ is the mass fraction of the fine structure, $\dot{m}^{*}$ is the specific mass flow rate through the fine structure, and * and bar represent the fine structure and average values, respectively. The first term on the right-hand side of equation 4 represents the turbulent mixing between the fine structures and the surroundings, while the second term represents the finite rate of reaction. The left-hand side represents the time evolution of species in the fine structures.
The corresponding equation for energy conservation in the fine structures is expressed as

$$
\begin{aligned}
\frac{\mathrm{d} T^{*}}{\mathrm{~d} t}= & -\frac{1}{C_{P}^{*}}\left(\frac{\dot{m}^{*}}{1-\chi \gamma^{*}} \sum_{m=1}^{M}\left(Y_{m}^{*} h_{m}^{*}-\bar{Y}_{m} \bar{h}_{m}\right)\right. \\
& \left.+\sum_{m=1}^{M} \frac{h_{m}^{*} \dot{\omega}_{m}{ }^{*} W_{m}}{\rho^{*}}\right)
\end{aligned}
$$

The specific mass flow rate through the fine structure can be expressed as

$$
\dot{m}^{*}=2.43(\varepsilon / v)^{1 / 2}
$$

where $v$ is the kinematic viscosity of the gas mixture and $\varepsilon$ is the turbulent energy dissipation. The mass fraction of the fine structures is given as

$$
\gamma^{*}=9.7\left(v \varepsilon / k^{2}\right)^{3 / 4}
$$

where $k$ represents the turbulent kinetic energy. The constants, 2.43 and 9.7, used in equations 6 and 7 are the same as those originally proposed by Magnussen [27].

The average density is related to the densities in the fine structures (denoted by *) and surrounding fluid (denoted by ${ }^{\circ}$ ) as

$$
1 / \bar{\rho}=\chi \gamma^{*} / \rho^{*}+\left(1-\chi \gamma^{*}\right) / \rho^{\circ}
$$

In this study, the value of $\chi$ is taken to be unity, since all species in the fine structures participate in chemical reactions. Although the governing equations are different from those of the original EDC model, the basic concept of the EDC model still holds. The mean reaction rate for species $m$ is determined by mixing between the fine structures and the surroundings:

$$
\bar{R}_{m}=\frac{\bar{\rho} \gamma^{*} \dot{m}^{*}}{\left(1-\gamma^{*}\right)}\left(Y_{m}^{*}-\bar{Y}_{m}\right)
$$

\section{Transition from the Chemical Ignition Model to the Modified EDC Model}

In most combustion systems, chemical ignition can be attributed to rapid radical growth and/or thermal runaway. Detailed chemistry is incorporated in the model to monitor radical concentrations and local temperatures at the grid level. Therefore, the combined effects of chemical and thermal ignition are accounted for in the proposed model. We postulate depletion of a certain amount of fuel will capture the rate of radical growth and/or thermal runaway. Therefore, the transition from the chemistry-only ignition model to the modified EDC model is determined on the basis of the value of the normalized residual fuel mass fraction.

Figure 1 is a schematic representing the range of fuel mass fractions that can be observed in a typical combustion system or computational cell. In particular, Fig. 1 represents the fuel methane. Here, $Y_{\mathrm{f}, \text { mix }}$ 
is the mass fraction of fuel in each cell when only mixing occurs (i.e., frozen chemistry), and $Y_{\mathrm{f}, \mathrm{eq}}$ is the mass fraction of fuel when the mixture is in equilibrium at the local cell temperature and fuel equivalence ratio. The actual fuel mass fraction in the cell has to exist between these two limiting values, where $Y_{\mathrm{f}, \text { mix }}$ is the upper limit and $Y_{\mathrm{f}, \mathrm{eq}}$ is the lower limit. In our work, we use a concept to define the transition between infinitely fast chemistry and frozen chemistry which is based on the parameter $\beta$, defined as:

$$
\beta=\left(Y_{\mathrm{f}, \text { mix }}-Y_{\mathrm{f}}\right) /\left(Y_{\mathrm{f}, \text { mix }}-Y_{\mathrm{f}, \mathrm{eq}}\right)
$$

Depending on the value of the transition parameter $\beta^{*}$, either the pure chemistry or the chemistry-mixing model (the modified EDC model) is used to determine the local reaction rate. In other words, based on the criteria:

$$
\left[\begin{array}{ccc}
\text { Modified } & \text { EDC } & \text { model }-\beta \geq \beta^{*} \\
\text { Chemical } & \text { ignition } & \text { model }-\beta<\beta^{*}
\end{array}\right]
$$

the submodels of model 2 are activated or deactivated. Here, $\beta^{*}$ is the criterion value for the transition parameter.

It is important to note the physical significance of the transition criterion. The transition parameter $\beta$ essentially indicates the extent of progress in a reaction cell. The limit of $\beta=0$ corresponds to very slow chemistry, where the time for mixing at the molecular level is orders of magnitude shorter than the time required for chemical kinetics to proceed. The limit of $\beta=1$ corresponds to infinitely fast chemistry, where mixing time scales are large and equilibrium kinetics are valid. For conditions where mixing and chemistry time scales are of the same order of magnitude $(1>\beta>0)$, the modified EDC model is used to define the combustion rate with simultaneous contributions from mixing and chemistry. Note that the transition parameter is not an indication of the degree of mixing at the molecular level nor an indication of the local level of turbulence.

\section{Experimental Work}

The experimental work of Naber et al. [6] was used for model validation. In their work, the authors determined ignition delay times for methane injected into a constant-volume chamber at high pressures and temperatures. The measurements for a density of $20.4 \mathrm{~kg} / \mathrm{m}^{3}$ and different temperatures are used for comparison.

The experimental vessel had a disk-shaped combustion chamber with a diameter of $114 \mathrm{~mm}$ and a width of $28.6 \mathrm{~mm}$. The chamber walls were kept at a constant temperature of $450 \mathrm{~K}$. The electronically controlled gaseous injector had a single orifice, $0.25 \mathrm{~mm}$ in diameter, which was oriented to inject fuel through the center of the chamber. The injection pressure was kept fixed at $20.7 \mathrm{MPa}$ and the injection duration was 11-13 ms. The temperature of the injected fuel was held constant at $450 \mathrm{~K}$.

Ignition delay was defined as the time it took for pressure in the bomb to increase $14 \mathrm{kPa}$ from the start of injection. The value of $14 \mathrm{kPa}$ corresponded to heat release by burning $0.33 \mathrm{mg}$ of fuel or $2.5 \%$ of the total mass of fuel injected. The measured pressure delay data was fit to the following ignition delay correlation [6]:

$$
\tau_{\mathrm{pd}}=\left\{C^{2}+\left[A\left(p / p_{\mathrm{o}}\right)^{-r} \exp \left(E_{\mathrm{a}} / \tilde{R} T\right)\right]^{2}\right\}^{1 / 2}
$$

where $C$ is a constant that represents physical mixing, $A$ is the Arrhenius constant (fuel-dependent), $p$ is the ambient pressure, $p_{\mathrm{o}}$ is the reference pressure (6.2 MPa), $r$ is the pressure correlation exponent (fuel-dependent), $E_{\mathrm{a}}$ is the Arrhenius apparent activation energy (fuel-dependent), $\tilde{R}$ is the universal gas constant, and $T$ is the core temperature of the ambient gas. The core temperature refers to the uniform temperature of approximately $90 \%$ of the volume of the bomb. Additional experimental details are described in Naber et al. [6].

\section{Numerical Simulations}

A non-uniform, Cartesian three-dimensional computational grid, with densification in the region near the injector, was used to describe domain dimensions of $11.4 \times 11.4 \times 2.86 \mathrm{~cm}^{3}$. The injector cell was located in the center of the computational domain and had a cross section of $0.3 \times 0.3 \mathrm{~mm}$. The injection velocity was obtained by multiplying a discharge coefficient $\left(C_{\mathrm{D}}=0.87\right)$, determined from experiments, to the isentropic injection velocity to account for choking effects at the injector. The mass flow rate based on the injection velocity, cross-sectional area of the orifice, and the critical pressure and temperature was found to be $1.2 \mathrm{~g} / \mathrm{s}$. A figure of the fuel injection profile used in the current work can be found in Agarwal and Assanis [1]. The calculated mass flow rate corresponds well to the experimental injection rate during the steady-state portion of injection, as $14 \mathrm{mg}$ of fuel was injected over a period of $11-13 \mathrm{~ms}$, leading to a mass flow rate between 1.1 and $1.27 \mathrm{~g} / \mathrm{s}$.

The internal energy in the injector cell was specified to simulate the cold fuel injected in the computational domain. To account for turbulence associated with the fuel injection, a value of turbulent kinetic energy corresponding to $1 \%$ of the injection velocity squared (based on the work of Papageorgakis and Assanis [22]) was added to the turbulent kinetic energy in the injection cell. A range of ambient temperatures from 1200 to $1500 \mathrm{~K}$ was simulated at an ambient density of $20.4 \mathrm{~kg} / \mathrm{m}^{3}$. 


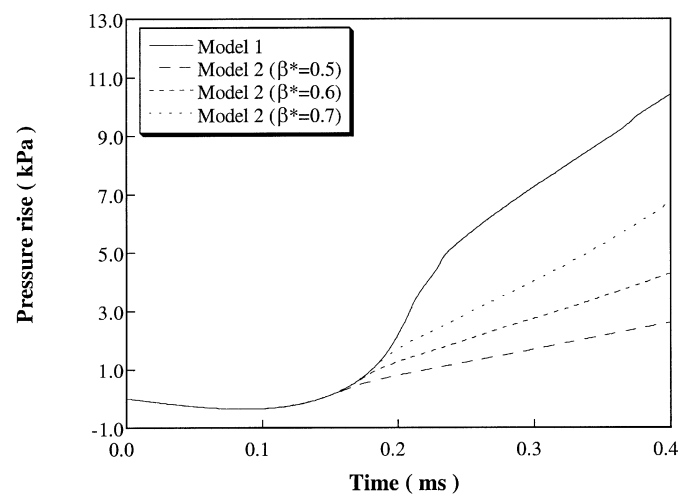

FIG. 2. Pressure rise predicted using models 1 and 2 with different transition criteria $\beta^{*}$ for an ambient temperature of $1400 \mathrm{~K}$.

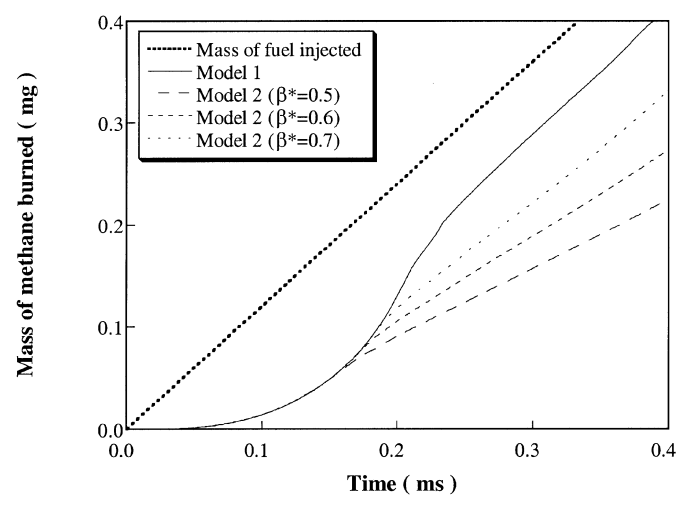

Fig. 3. Mass of methane burned, computed using different values of the transition criterion $\beta^{*}$, for an ambient temperature of $1400 \mathrm{~K}$.

\section{Results and Discussion}

The simulation results of models 1 and 2 for volume-averaged pressure as a function of time for an ambient temperature of $1400 \mathrm{~K}$ are shown in Fig. 2. The chemistry-only model results (model 1) show two interesting features: (1) cylinder pressure decreases in the early phase of ignition; (2) the rate of pressure rise decreases after the initial rapid increase in pressure. The initial pressure drop results from the endothermic process of fuel oxidation. The decrease in the slope (which occurs around $0.24 \mathrm{~ms}$ ) after the initial rapid pressure rise (attributed to combustion of any fuel in the cylinder which has time to become premixed) represents the fact that the rate of methane burning is restricted by the rate of fuel injection. This is easily confirmed when the slope of the mass of methane burned is compared with that of mass of methane injected (see Fig. 3).
The slopes of model 1 and the mass of methane injected are the same after $\sim 0.24 \mathrm{~ms}$, and they represent the upper limit in the fuel consumption rate, where subgrid scale mixing is neglected.

Figure 2 also shows the effects of mixing on prediction of ignition and how the transition criterion affects the results of the model 2 . The effects of mixing appear near the end of the induction period. The slopes of the pressure profiles predicted using the proposed model are different from that predicted using the chemistry-only model, indicating reaction rates are affected by the turbulent fuel/air mixing rate as well as chemical reaction rate. Values for $\beta^{*}<0.5$ allow model 2 to include mixing effects too early, to the point that mixing effects retard or prevent flame development. On the other hand, the slope for $\beta^{*}=0.7$ at later times is almost identical with the results of the chemistry-only model (model 1). Therefore, appropriate criterion values for the transition parameter are $\beta^{*}$ between 0.5 and 0.7 . The optimal value for $\beta^{*}$ is determined via comparison with the experimental results, and is discussed further below.

The simulation results for the mass of methane burned as a function of time are shown in Fig. 3. The general characteristics are the same as those of Fig. 2, with the primary difference that there is no decrease in the mass of methane burned at early times. The differences between the models are smaller than those of the pressure rise; however, the effects of mixing are still readily apparent in Fig. 3. In the early phase of ignition, until time reaches $0.20 \mathrm{~ms}$, the reaction rates depend only on chemical kinetics, a finding which can be deduced from the identical results predicted by both models. Subsequently, while ignition is still occurring, mixing affects the reaction rates and results in different rates of fuel consumption.

Two ignition criteria were considered in this work based on the pressure and fuel profiles: the time for an increase in pressure of $11 \mathrm{kPa}$ and the time to consume $0.33 \mathrm{mg}$ of fuel. The value of $11 \mathrm{kPa}$ is $\pi / 4$ times the value of $14 \mathrm{kPa}$ used in the experimental study. Because the computations were performed using a grid with a volume $4 / \pi$ times larger than the experimental chamber volume, the first law dictates that the average computational pressure rise should be $\pi / 4$ times the experimental value for the same heat release. However, the simulation results did not agree with the experimental data when the $11-\mathrm{kPa}$ pressure rise was used as the ignition criterion. On the other hand, simulation results based on the fuel consumption criterion showed excellent agreement with experimental data. Therefore, all ignition delay times presented in this study were based on the criterion of $0.33 \mathrm{mg}$ of fuel burned.

Five sets of ignition delay time are plotted in $\mathrm{Ar}$ rhenius coordinates $(\ln (\tau)$ versus $1 / T)$ in Fig. 4. For comparison purposes, the empirical correlation of 


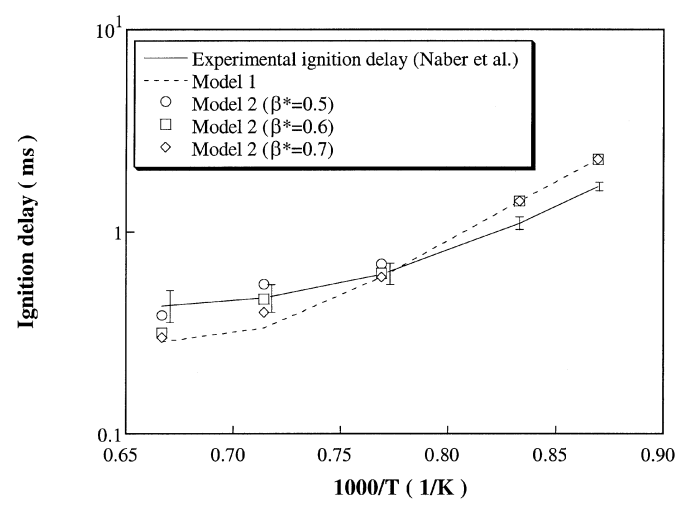

FIG. 4. Comparison of ignition delay times derived from the current work with the experimental correlation of $\mathrm{Na}-$ ber et al. [6] (model $1=$ pure chemistry approach; model $2=$ combined chemistry and mixing approach). The error bars represent the uncertainty in the experimental measurements and are offset for clarity.

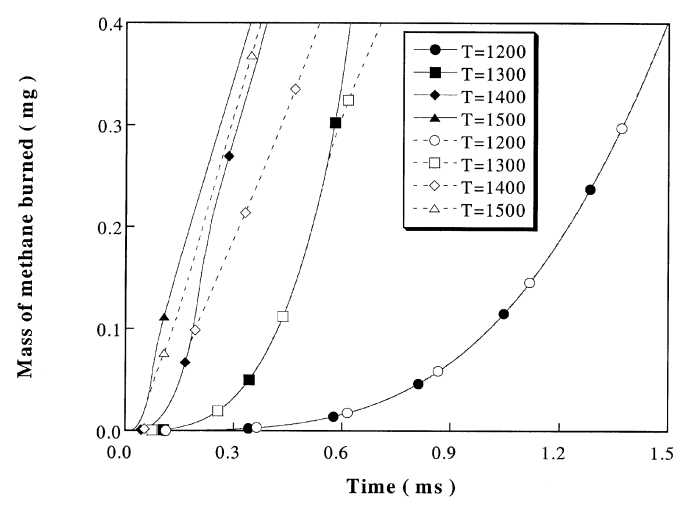

FIG. 5. Mass of methane burned for different temperatures predicted by model 1 (solid lines and solid symbols) and model 2 (dashed lines and open symbols) for a criterion transition value of $\beta^{*}=0.6$.

Naber et al. (equation 12 [6]) is also indicated in Fig. 4 by the solid line. The dashed line indicates the predicted values using the chemistry-only model (model 1), in which the reaction rates depend on chemical kinetics [1]. The open symbols are the results of model 2 simulations for $\beta^{*}$ values of $0.5,0.6$, and 0.7 . The predictions were performed for temperatures ranging between 1200 and $1500 \mathrm{~K}$, which are typical for methane gas ignition. Results of the new model show considerable improvement in the ignition delay predictions at high temperatures $(T>$ $1300 \mathrm{~K}$ ) compared with those of the chemistry-only model. Naber et al. [6] reported that the ignition delay approached the physical mixing delay for temperatures greater than $1400 \mathrm{~K}$. The results of the current numerical simulations support that conclusion and demonstrate that fuel/air mixing plays an important role in determining the reaction rate during the ignition period at high temperatures.

To further investigate the effects of temperature, several profiles of the mass of methane burned are presented for models 1 and 2 in Fig. 5. The criterion value used in model 2 is $\beta^{*}=0.6$. For an ambient temperature of $1200 \mathrm{~K}$, the predicted mass of methane burned with models 1 and 2 are identical. We can conclude that chemical kinetics dominate at low temperatures, as expected. The first small differences between models 1 and 2 are detected in the results for $T=1300 \mathrm{~K}$. The difference appears shortly before the mass of methane burned reaches $0.3 \mathrm{mg}$, which agrees with experimental observations [6], when the fuel-consumption-based ignition criterion is used. At temperatures of 1400 and $1500 \mathrm{~K}$, the differences between the results of models 1 and 2 are apparent at earlier times. This suggests that the premixed combustion phase, which is chemistry controlled, is short, and turbulent mixing participates in determining the reaction rates from early times. The results are in accordance with the findings of Naber et al. [6], who concluded that for $T>$ $1400 \mathrm{~K}$, the ignition delay approaches a limiting value and physical mixing is dominant.

\section{Summary and Conclusions}

The current work presents an improved modeling approach with strong physical basis for predicting reaction rates during ignition delay which accounts for both chemistry and mixing in direct-injection, stratified charge combustion systems. The EDC model, which determines reaction rates based on interaction between chemistry and mixing, has been modified to include transient effects. The modified EDC model is interfaced with a chemistry-only model using a physically based transition parameter $\beta$. The simulation results employing the new transition and chemistry-mixing submodels agree well with experimental data. The results of the current work also identify combustion conditions where physical mixing and detailed chemistry both significantly affect ignition delay. In particular, the computational results for temperatures above $1300 \mathrm{~K}$ show mixing is important at these conditions, which is consistent with experimental observations.

The sensitivity of the simulations to the transition parameter was studied and an optimal value of $\beta^{*}=$ 0.6 was identified. The primary factor affecting the determination of $\beta^{*}$ is the fuel characteristics. For example, higher hydrocarbons such as iso-octane show different fuel oxidation and ignition characteristics compared to methane. Therefore, the transition parameter used here (based on fuel consumption) may need to be modified for alternative fuel applications. 
Future work includes further evaluation of $\beta^{*}$ for simulation conditions including additional fuels, turbulent intensity, and chamber geometries. Note that the approach used in this work is robust and can be used to evaluate the relative effects of chemistry and mixing for a broad range of engine fuel/air preparation strategies, ranging from fully premixed (e.g., homogeneous charge compression ignition combustion) to stratified charge engine technologies.

\section{Acknowledgments}

The authors gratefully acknowledge the contributions of Dr. Apoorva Agarwal. This work has been supported in part by the Automotive Research Center (ARC), a consortium of eight universities directed by the University of Michigan. The ARC is funded by the National Automotive Center located within the Tank-Automotive Research, Development and Engineering Center in Warren, Michigan.

\section{REFERENCES}

1. Agarwal, A., and Assanis, D. N., Combust. Sci. Technol. 163:177 (2001).

2. Jennings, M. J., and Jeske, F. R., ASME Trans. J. Eng. Gas Turb. Power 116:799 (1994).

3. Niioka, T., Proc. Combust. Inst. 18:1807 (1981).

4. Sato, J., Konishi, K., Okada, H., and Niioka, T., Proc. Combust. Inst. 21:695 (1986).

5. Mastorakos, E., Baritaud, T. A., and Poinsot, T. J., Combust. Flame 109:198 (1997).

6. Naber, J. D., Siebers, D. L., Caton, J. A., Westbrook, C. K., and Di Julio, S. S., SAE paper 94-2034.

7. Henein, N. A., and Bolt, J., SAE paper 69-0252.

8. Aggarwal, S. K., and Sirignano, W. A., Proc. Combust. Inst. 20:1773 (1984).

9. Takahashi, F., Mizomoto, M., Ikai, S., and Futaki, N., Proc. Combust. Inst. 20:295 (1984).

10. Liñán, A., and Williams, F. A., Combust. Flame 95:3146 (1993).
11. Emerson, R. G., and Rutland, C. J., paper 99-ICE-167, 1999 Spring Technical Conference ASME, ICE-Vol. 32-1, 1999.

12. Dillies, B., Ducamin, A., Lebrere, L., and Neveu, F., SAE paper 97-0880.

13. Kong, S.-C., Han, Z., and Reitz, R. D., SAE paper 950278.

14. Amsden, A. A., Los Alamos National Laboratory report LA-13313-MS, 1997.

15. Kong, S.-C., and Reitz, R. D., paper 2000-ICE-306, 2000 ICE Fall Technical Conference ASME, ICE-Vol. 35-1, 2000.

16. Sloane, T. M., and Ronney, P. D., Combust. Sci. Technol. 88:1 (1992).

17. Mulholland, J. A., Sarofim, A. F., and Beer, J. M., Combust. Sci. Technol. 87:139 (1992).

18. Varatharajan, B., and Williams, F. A., Combust. Flame 121:551 (2000).

19. Glassman, I., Combustion, Academic Press, San Diego, 1996.

20. Williams, F. A., Combustion Theory, 2nd ed., AddisonWesley, Redwood City, CA, 1985.

21. Fotache, C. G., Wang, H., and Law, C. K., Combust. Flame 117:777 (1999).

22. Papageorgakis, G. C., and Assanis, D. N., SAE paper 98-0135.

23. Kee, R. J., Rupley, F. M., and Miller, J. A., Sandia report SAND89-8009B.

24. McBrid, B. J., and Gordon, S., NASA Reference Publication 1311 June, National Aeronautics and Space Administration, Lewis Research Center, Cleveland, Ohio, 1996.

25. Frenklach, M., Wang H., Goldenber, M., Bowman, C. T., Hanson, R. K., Smith, G. P., Golden, D. M., Gardiner, W. C., and Lissianski, V., Gas Research Institute report GRI-95/0058, 1995.

26. Hindmarsh, A. C., in Scientific Computing (R. S. Stepleman et al., eds.), IMACS Trans. on Scientific Computation, North-Holland, Amsterdam, 1983, pp. 55.

27. Magnussen, B. F., in XI Task Leaders Meeting: Energy Conservation in Combustion, IEA, 1989, p. 248.

\section{COMMENTS}

Dimitrios Kyritsis, Yale University, USA. I wonder what range of Damköhler $(D a)$ numbers is valid for your model? Can it be that the disagreement with experimental data at higher rotational speeds is due to Da effects?

Author's Reply. The chemistry-dependent ignition model is valid for low Da. As combustion proceeds, the characteristic time for chemical reaction decreases and $D a$ increases. Therefore the assumption of low $D a$ is no longer valid. Using the modified EDC model, both chemistry and turbulent mixing effects are simultaneously accounted for, thus making the modeling approach valid for both low and high values of $D a$. The difference at high temperatures between the experimental data and the ignition model that only incorporates detailed chemistry is likely due to mixing effects.

Salah S. Ibrahim, Loughborouh University, UK. Your modified combustion model assumes that the time scale for chemical reaction is taken to be equal to that of the turbulence mixing time scale (i.e., $\propto \varepsilon / \kappa)$ ). Can you comment on the validation of this assumption for engine calculations?

Author's Reply. As mentioned in our response to the first question the modified combustion model accounts for a 
range of Damköhler numbers. When $D a<1$, the chemistry dependent ignition model is used. When $D a \geq 1$, the modified EDC model is used to determine the reaction rate using contributions from both the detailed chemical mechanism and the turbulent mixing. The exact transition between the two submodels is determined at each node at each point in time using the transition parameter $\beta$. We feel the transition parameter $\beta$ (based on the fuel consumption) is a good indication of when thermal and/or radical runaway for the $\mathrm{CH} 4$ system has been achieved and the characteristic time for chemical reaction is therefore small (i.e., $D a \sim 1$ ).

John Abraham, Purdue University, USA. The authors may want to clarify the use of the terminology "pure chemistry model" (model 1). The effects of mixing are included in both models as Reynolds-averaged equations are solved for the species. Furthermore, the authors may want to comment on the validity of employing the chemical kinetic mechanism in the context of solving the Reynolds-averaged equations for turbulent flows.

Author's Reply. The phrase pure chemistry was meant to indicate the approach used to model the ignition phenomena. Clearly mixing effects at the grid level (large scale) are included in both models. However, model 2, which includes the modified EDC model and detailed chemistry, is a means to simulate molecular mixing at the subgrid level (i.e., small scale mixing) where reactions occur in a small scale-mixing zone.

The kinetic mechanism is not used directly in solving the Reynolds-averaged equations. The detailed chemistry is used in the determination of the reaction rate in each cell. The Reynolds-averaged equations are then used to determine the distribution of species. 